\title{
ERRATUM
}

\section{Neuropsychological Recovery Trajectories in Moderate to \\ Severe Traumatic Brain Injury: Influence of Patient \\ Characteristics and Diffuse Axonal Injury - Erratum}

Amanda R. Rabinowitz, Tessa Hart, John Whyte, And Junghoon Kim

doi: 10.1017/S1355617717000996. Published online by Cambridge University Press on 16 October 2017.

The paper by Rabinowitz et al. (2017) has errors in the article that should be brought to the attention of readers. On page 237, the authors' affiliations are given incorrectly.

\section{Errant copy}

Amanda R. Rabinowitz, ${ }^{1,2}$ Tessa Hart, ${ }^{1}$ John Whyte, ${ }^{1}$ and Junghoon Kim ${ }^{1}$

${ }^{1}$ Moss Rehabilitation Research Institute, Elkins Park, Pennsylvania

${ }^{2}$ CUNY School of Medicine, The City College of New York, New York, New York

\section{Correct copy}

Amanda R. Rabinowitz, ${ }^{1}$ Tessa Hart, ${ }^{1}$ John Whyte, ${ }^{1}$ and Junghoon Kim ${ }^{1,2}$

${ }^{1}$ Moss Rehabilitation Research Institute, Elkins Park, Pennsylvania

${ }^{2}$ CUNY School of Medicine, The City College of New York, New York, New York

Cambridge University Press and the Editor regret the inconvenience that this error may have caused. 